\title{
Evolutionary Optimization Algorithms and Machine Learning for Engineering Applications
}

\author{
Peter Poposki \\ Faculty of Computer Sciences, Saint Petersburg State University Russia
}

\begin{abstract}
The manuscript investigates the novel evolutionary optimization algorithms and machine learning for engineering applications. Several case studies and comparative analysis are discussed.
\end{abstract}

\section{References:}

Khan, H., Wahab, F., Hussain, S., Khan, S., Rashid, M. Multi-object optimization of Navy-blue anodic oxidation via response surface models assisted with statistical and machine learning techniques (2022) Chemosphere.

Sui, Z., Sui, Y., Wu, W. Multi-objective optimization of a microchannel membrane-based absorber with inclined grooves based on CFD and machine learning (2022) Energy

Zhang, H., He, B., Lu, G., Zhu, Y. A simulation and machine learning based optimization method for integrated pedestrian facilities planning and staff assignment problem in the multi-mode rail transit transfer station (2022) Simulation Modelling Practice and Theory.

Mosavi, Amir, and Atieh Vaezipour. "Reactive search optimization; application to multiobjective optimization problems." Applied Mathematics 3.10A (2012): 1572-1582.

Raat, W., Smeets, M., Henrard, S., Aertgeerts, B., Penders, J., Droogne, W., Mullens, W., Janssens, S., Vaes, B. Machine learning optimization of an electronic health record audit for heart failure in primary care (2022) ESC Heart Failure

Feng, Q., Maier, W., Stehle, T., Möhring, H.-C. Optimization of a clamping concept based on machine learning (2022) Production Engineering.

Mosavi, Amir. "The Large Scale System of Multiple Criteria Decision Making; Pre-processing." IFAC Proceedings Volumes 43.8 (2010): 354-359.

Fernández, M., Fritzen, F., Weeger, O. Material modeling for parametric, anisotropic finite strain hyperelasticity based on machine learning with application in optimization of metamaterials (2022) International Journal for Numerical Methods in Engineering.

Liu, J.-J., Liu, J.-C. Permeability Predictions for Tight Sandstone Reservoir Using Explainable Machine Learning and Particle Swarm Optimization (2022) Geofluids.

Kalbasi, Rasool, et al. "Finding the best station in Belgium to use residential-scale solar heating, one-year dynamic simulation with considering all system losses: economic analysis of using ETSW." Sustainable Energy Technologies and Assessments 45 (2021): 101097.

Venkata Rao, K., Parimi, S., Suvarna Raju, L., Suresh, G. Modelling and optimization of weld bead geometry in robotic gas metal arc-based additive manufacturing using machine learning, finite-element modelling and graph theory and matrix approach (2022) Soft Computing, . 
Liu, Q., Zeng, Z., Jin, Y. Distributed machine learning, optimization and applications (2022) Neurocomputing.

Qasem, Sultan Noman, et al. "Estimating daily dew point temperature using machine learning algorithms." Water 11.3 (2019): 582.

Mosavi, A. (2010). Multiple criteria decision-making preprocessing using data mining tools. arXiv preprint arXiv:1004.3258.

Karballaeezadeh, N., Mohammadzadeh S, D., Shamshirband, S., Hajikhodaverdikhan, P., Mosavi, A., \& Chau, K. W. (2019). Prediction of remaining service life of pavement using an optimized support vector machine (case study of Semnan-Firuzkuh road). Engineering Applications of Computational Fluid Mechanics, 13(1), 188-198.

Mosavi, A., \& Rabczuk, T. (2017). Learning and intelligent optimization for material design innovation. In International Conference on Learning and Intelligent Optimization (pp. 358-363). Springer, Cham.

Mosavi, A., \& Rabczuk, T. (2017). Learning and intelligent optimization for material design innovation. In 11th International Conference on Learning and Intelligent Optimization, LION 2017 (pp. 358-363). Springer Verlag.

Samadianfard, Saeed, et al. "Wind speed prediction using a hybrid model of the multi-layer perceptron and whale optimization algorithm." Energy Reports 6 (2020): 1147-1159.

Shabani, Sevda, et al. "Modeling pan evaporation using Gaussian process regression K-nearest neighbors random forest and support vector machines; comparative analysis." Atmosphere 11.1 (2020): 66.

Dineva, Adrienn, et al. "Review of soft computing models in design and control of rotating electrical machines." Energies 12.6 (2019): 1049.

Torabi, Mehrnoosh, et al. "A hybrid machine learning approach for daily prediction of solar radiation." International Conference on Global Research and Education. Springer, Cham, 2018. 